\title{
Special meeting report
}

\section{CaPCURE's fourth meeting: the cutting edge of current prostate cancer research}

\author{
J Naitoh \& A Belldegrun \\ Department of Urology, UCLA School of Medicine, Box 951738, Los Angeles, \\ CA 90095-1738, USA
}

\section{Introduction}

Between September 4-7, 1997, over two hundred clinicians and scientists gathered for the Fourth Annual Association for the Cure of Cancer of the Prostate (CaP CURE) Scientific Retreat held at the Hyatt Regency Resort in Lake Tahoe, Nevada. During the three day meeting, over 100 presentations were delivered, covering the state of the art in prostate cancer treatment and research. Organized by Stuart Holden, MD, the Medical Director of CaP CURE, and Richard N. Atkins, MD, the Chief Operating Officer of CaP CURE, the Scientific Retreat was designed to gather 'the vanguard of prostate cancer research,' to provide an informal environment for the exchange of ideas, and also to present an update of ongoing CaP CURE sponsored research.

Over the last four years, the Santa Monica based, nonprofit CaP CURE organization has allocated over $\$ 35$ million to 270 projects worldwide, with the goals of encouraging basic prostate cancer research, providing for the rapid translation of this research from the laboratory setting into the clinical setting, and devising curative therapies for advanced prostate cancer. CaP CURE is currently second only to the National Cancer Institute in

Correspondence: Dr Arie Belldegrun, c/o Virginia Baeta, Dept. of Urology, UCLA School of Medicine, BOX 951738, Los Angeles, CA 90095-1738, USA terms of funding prostate cancer research, and is the largest private source for research funding.

This year's meeting was intended to review the advances made by $\mathrm{CaP}$ CURE researchers over the last year, and was divided into 11 sessions, covering molecular biology, experimental therapeutics, angiogenesis, vaccine therapy, clinical therapies, apoptosis, androgen receptors, genetics, clinical trials, radiation and imaging, and nutrition. There were numerous advances reported in each of these sessions ranging from the molecular biology of the androgen receptor to the development of novel chemotherapeutic agents that are activated by the protease activity of PSA. This review is by no means comprehensive, but it intends to update the practicing clinician with the new and exciting directions in prostate cancer research and therapy.

\section{Gene discovery}

One of the most significant programs created by $\mathrm{CaP}$ CURE was the Genetics Consortium, which combines the unique resources of UCLA, the University of Washington, and the Washington University School of Medicine in St. Louis. The main goals of this consortium are to identify families with hereditary prostate cancer, create tissue and serum banks of specimens collected from hereditary and sporadic cases, use these specimens to look for prostate cancer genes, and to create new animal models for the 
study of prostate cancer. At this time, the Consortium has had a moderate degree of success in achieving all of these aims.

With consortium assistance, Charles Sawyers, MD, Rob Reiter, MD, and Owen Witte, MD, of the UCLA School of Medicine, have been able to generate five new mouse xenograft models for prostate cancer. Out of the five tumor models and cell lines they have developed so far, the LAPC4 line has proven to be particularly interesting, since it has a propensity to develop metastasis upon orthotopic injection into SCID mice and can induce osteoblastic lesions upon injection into the bone marrow. Study of such a model not only allows for the identification of the genetic changes that cancer cells undergo as they progress, but it will also allow for the testing of new treatments against androgen dependent and independent prostate cancer.

Additionally, Consortium researchers have begun to screen tissue from the hundreds of hereditary prostate cancer cases that were collected by Paul Lange, MD, of the University of Washington, and William Catalona, MD, of the Washington University School of Medicine. In combination with the microchip technology created by AFFYMETRIX, rapid throughput screening of thousands of candidate prostate cancer genes has been performed from these samples. Unfortunately, as seen by Consortium researchers, the identification of a single gene that is involved in all cases of prostate cancer has not been simple and may not even be possible, simply because such a master gene may not exist. It's probable that the prostate cancer phenotype arises from multiple, independent mutations in receptor proteins, in signal transduction enzymes, in co-activating proteins, and in nuclear binding proteins.

This heterogeneity of the prostate cancer genotype is reflected in the findings of gene identification studies that have been done so far. Studies performed at Johns Hopkins and by the University of Washington have had conflicting results regarding the location of the hereditary prostate cancer gene. Last year, researchers from Johns Hopkins University reported the chromosomal location of a putative familial prostate cancer gene (HPC 1). While the actual gene has yet to be identified, studies derived from the familial cohorts collected at the Johns Hopkins Hereditary Gene Project showed strong evidence for linkage of a gene at chromosome site 1q24-25 to the occurrence of prostate cancer based on pedigree analysis.

In comparison, through the work of Janet Stanford, Ph.D., Elaine Ostrander, Ph.D., Leroy Hood, MD, Ph.D., and others from the University of Washington and the Fred C. Hutchinson Cancer Research Center, no significant linkage for a prostate cancer gene was found at the 1q24-25 site in the familial cases that were identified by the Consortium. Instead, chromosome 1p appeared to be the locus of a hereditary prostate cancer gene. It's probable that demographic differences between the two populations can explain the differences between the two groups. Again, these results indicate that the genotype of prostate cancer is probably going to be heterogeneous with multiple genetic lesions involved in the initiation and growth of prostate cancer.
However, one of the most interesting genetic changes that is associated with prostate cancer involves telomeric stabilization. The telomere is located at the ends of each chromosome and appears to work as a mitotic clock, with each mitotic division resulting in a shortening of the telomere. Eventually, when the telomere hits a critical length, 'cell crisis' occurs, and the cell enters a pathway of programmed cell death. In contrast, tumor cells have the enzyme telomerase, which stabilizes the telomere and prevents 'crisis' from ever occurring. Thus far, over 95\% of tumor specimens studied express telomerase, implying that there is a critical role for this enzyme in cellular immortalization. Additionally, since this enzyme is not expressed in any other tissue in the body except the testis, therapy targeting telomerase will have significant potential to provide for effective tumor killing while causing minimal toxicity to normal cells.

\section{Tumor markers}

Prostate Specific Antigen (PSA), despite the fact that it is the most powerful tumor marker available in oncology today, has many significant inherent limitations. Due to the limited specificity of PSA and its inability to predict tumor stage or the biologic potential of the tumor, the search for better diagnostic and prognostic markers has continued.

David Ralph, Ph.D., of Urocor, Inc., Robert Reiter, MD, of the UCLA School of Medicine, and Marion J. Bussemakers, Ph.D., of the University Hospital, Nijmegen, have each identified new, putatively prostate cancer specific genes. The genes described by Dr. Reiter and Dr. Bussemakers appeared to be particularly interesting with regards to their potential diagnostic, prognostic, and therapeutic implications.

Dr. Bussemakers described a novel prostate tumor marker called DD3. While the structure and function of DD3 are unknown, DD3 appeared to be over-expressed in prostate cancer, while it was absent in $\mathrm{BPH}$ and in all other normal tissues studied. Additionally, DD3 expression appeared to correlate with higher tumor grade, higher pathological stage, and to the occurrence of distant metastasis. While DD3 has potential utility as a diagnostic, prognostic, staging, and therapeutic agent, more work is needed to characterize expression of the protein, since antibodies that can recognize the DD3 gene product have yet to be developed.

Dr. Reiter and his colleagues also identified a gene (called Prostate Stem Cell Antigen, or PSCA) that was expressed highly in both the androgen resistant and the androgen sensitive clones of the LAPC4cell line. PSCA appears to be a cell surface protein whose expression is prostate specific: while it can be found in basal cells and carcinomatous glands, expression was absent in all other human tissues except in the placenta. Due to its prostate specific expression, and due to its localized expression on the cell surface, both diagnostic and therapeutic applications may arise following further evaluation of this gene and gene product. 
One other interesting hypothesis that arose from Dr. Reiter's study of the LAPC 4 line involved an analysis of the phenotype changes that occur as a cell progresses from the benign, to the malignant, and finally to the hormone refractory state. This progression of prostate call cytodifferentiation was further elaborated upon by Alvin Y. Liu, Ph.D., of the University of Washington. In his presentation, he described the basal cell-stromal cell interactions that are needed for the prostate cell to undergo a phenotypic progression from basal cell to lumenal cell (Figure 1). Additionally, given that late prostate cancers appear to express a phenotype that is very similar to that of normal basal cells, it has been hypothesized that prostate cancer actually originates from these basal cells, and that the study of the stromal cellcancer cell interaction, along with the pattern of gene expression in basal cells and prostate cancer cells, may be able to shed new light into the molecular origins of prostate cancer.

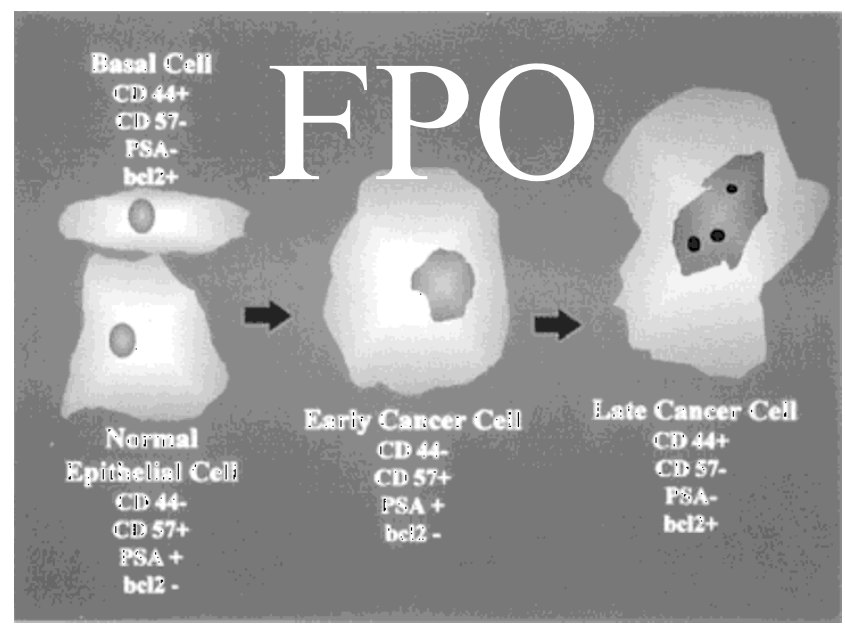

Figure 1. The phenotypic progression of prostate glandular cells, from basal cell to epithelial cell to carcinoma. Late cancers express a phenotype that is very similar to that of normal basal cells.

\section{Apoptosis}

Since failure of normal apototic mechanisms has been implicated in the etiology of both the initiation of prostate cancer and the occurrence of the androgen independent state, a significant volume of work was presented regarding the defects in the intracellular pathways that normally induce prostate cell apoptosis. Multiple inhibitors of apoptosis were described, including calreticulin (Zhou Wang, Ph.D., of Northwestern University) and Bax Inhibitor 1 (John Reed, MD, Ph.D., of the Burnham Institute). It is hoped that therapies directed against these apoptosis inhibitors will be effective and have minimal toxicity, since the regulation of apotosis in normal cells should be unaffected.
However, the most interesting treatment approaches targeting apotosis were described by Charles Myers, Jr., MD, of the University of Virginia, and by Ralph Buttyan, Ph.D., of Columbia University. Since there is a known correlation between high dietary fat to prostate cancer growth and progression, and since excess levels of 5hydroxyeicosanoids have been shown to protect cells from apoptotic stimuli, Dr. Myers studied the metabolic pathway for fatty acids in prostate cancer cell lines. He found excess levels of 5-hydroxyeicosanoids in prostate cancer cells, and further discovered that treatment of prostate cancer cell lines with a 5-lipoxygenase inhibitor (MK 886) resulted in decreased cellular levels of the 5hydroxyeicosanoids and rapid initiation of apoptosis. Furthermore, this effect was independent of bcl-2 gene expression and was blocked by the exogenous addition of 5-hydroxyeicosanoids to the cell cultures. Thus, the enzyme 5-lipoxygenase appears to play an important role in blocking apoptosis and therefore may represent a good target for therapy.

Another approach that can restore normal apoptotic pathways was discussed by Ralph Buttyan, Ph.D., of Columbia University. By creating an anti-bcl2 mRNA ribozyme (which provides for the specific degradation of bcl-2 mRNA), lipofectin transfer of the protein to LnCAP cell lines resulted in an $80 \%$ reduction in bcl-2 mRNA and the subsequent induction of apoptotic cell death. While this approach is promising based on in vitro data, further in vivo studies will have to be performed as soon as an efficient delivery vector can be constructed for this ribozyme. This therapy may also be ineffective in the many advanced tumors that are negative for bcl-2 expression.

\section{Angiogenesis}

Over the last year, many advances were made by $\mathrm{CaP}$ CURE investigators in the field of tumor angiogenesis, including the development of new treatment strategies that will destroy the new blood vessels that a tumor needs to grow. Anti-angiogenic therapy has significant appeal for several reasons: tumors cannot grow without a blood supply, tumor vessels may have an unique antigen profile that can make them susceptible to attack, and the therapy conceivably would not result in the development of resistance over time, since the non-cancerous vascular endothelial cells involved in angiogenesis should still respond normally to regulatory signals.

One of the more exciting approaches targeting tumor angiogenesis was described by Bruce Zetter, Ph.D., of Harvard Medical School. By using nude mice carrying implanted lung tumors, his group was able to show that treatment with the angiogenesis inhibitor endostatin resulted in significant regression of the tumors. Furthermore, multiple cycles of endostatin not only resulted in the reliable suppression of tumor growth, but also appeared to work without any sign that resistance to the treatment would occur with serial dosages or prolonged treatment. Additionally, while microscopic evidence of tumor persisted even after long term therapy 
with endostatin, after six cycles of treatment, the tumor failed to regrow following cessation of therapy.

Another anti-angiogenic treatment regimen involves the use of interferon beta, as reported by Isaiah Fidler, DVM, Ph.D., of the MD Anderson Cancer Center. Interferon beta levels are decreased in prostate cancer, with normal levels of interferon beta having multiple tissue effects including macrophage activation, inhibition of angiogenesis, and induction of apoptosis. Restoration of interferon beta levels via transfection of the gene into PC3 cells resulted in decreased tumorigenicity of the cell line, decreased production of FGF, decreased intratumoral angiogenesis, enhanced macrophage infiltration, and decreased metastatic potential of transfected tumor cells in nude mice. Due to these promising results, it's anticipated that clinical trials using interferon beta in prostate cancer will begin soon.

Besides these agents, human phase I/II trials have already been initiated using the anti-angiogenic compounds TNP 470 and thalidomide. Unfortunately, while efficacy data for TNP 470 is not available, William Figg, Ph.D., of the National Cancer Institute, showed that thalidomide had only limited efficacy in patients with hormone refractory disease. While responses appeared to be relatively durable and side effects were minimal, most patients eventually failed thalidomide therapy due to the progression of their boney metastasis, suggesting that angiogenic factors may not be required to promote the growth of tumors in the bone marrow. Thus, despite the promise of anti-angiogenic therapy, this early optimism for its role must be tempered by the fact that angiogenesis may be irrelevant for tumor growth in locations such as the bone marrow where tumor cells can exist in an oxygen and nutrition rich environment without the need for neovascularity.

\section{Gene therapy}

Multiple investigative sites have begun programs using gene therapy for prostate cancer, using genes that result in self destruction of the tumor, genes that result in the expression of antigenic proteins in the tumor cells, or genes that produce cytokines and stimulate the immune system to fight the cancer.

The most important clinical gene therapy data was presented by Peter Scardino, MD, of the Baylor College of Medicine. Using an attenuated adenovirus carrying the thymidine kinase gene, intratumoral injections of the vector were done in 18 patients. No mechanism for tumor specific expression was used beyond intraprostatic injection. Interestingly, a dose dependent response rate to the treatments was seen; at dose levels of between $10^{9}-10^{11}$ viral particles, $20-25 \%$ of patients had measurable declines in their PSA levels. Unfortunately, increasing the viral vector dose also resulted in increased toxicity, with the most recently treated patient (who was treated with the highest dose level) having grade IV hematologic toxicity and hepatic toxicity, possibly attributable to the nonspecific effects of this gene vector in multiple cell types.
In contrast, many other centers have focused on the development of prostate cancer specific gene treatment. Jonathan Simons, MD, and Ronald Rodriguez, MD, of Johns Hopkins Oncology Center, Arie Belldegrun, MD, of UCLA, Leland Chung, Ph.D., of the University of Virginia, and Jan Trapman, Ph.D., of Erasmus University, have all independently tried to use the PSA promoter sequence to confer target specificity to an attached suicide gene. Most investigators have reported promising results that used the PSA promoter in tandem with suicide genes such as diphtheria toxin or thymidine kinase. While questions remain regarding which promoter variation has the most specific activity, which vector will provide the safest and most efficient transfection of the gene, and which toxin gene should be attached to the promoter, multiple phase I clinical trials using these PSA promoter driven vectors will soon begin.

Additionally, Dr. Chung described the behavior of the osteocalcin promoter which may also be able to provide tumor specificity to suicide gene therapy. The potential advantage of this gene promoter is the high level of osteocalcin gene activity in bone stroma; since prostate cancer has a propensity to spread to the bone, osteocalcin driven gene therapy may represent a logical approach for patients with skeletal metastasis.

\section{Advances in immunotherapy}

Although prostate cancer has not traditionally been considered to be an immunologic tumor, many advances have been made to augment the immune response against prostate cancer cells. In general, the most promising approaches involve either the ex vivo creation of tumor vaccines, or the in vivo attempts to generate an immunologic response by the administration of antitumor antibodies or by transfecting cytokine genes directly into the tumor.

An exciting method to induce an antitumor immune response currently involves the use of dendritic cells. $\mathrm{H}$. Kim Lyerly, MD, of Duke University, and Gerald Murphy, MD, of the Pacific Northwest Cancer Foundation each presented pre-clinical and clinical data regarding the use of dendritic cell driven immunotherapy for prostate cancer. Thus far, while minimal efficacy data is available, the treatments have been well tolerated by the patients to date.

Norman Greenberg, Ph.D., of the Baylor College of Medicine, attempted to bypass the need for dendritic cell antigen presentation by transfecting the B7 gene directly into tumor cells, which should allow for the direct activation of CD4 and CD8 lymphocytes to specifically kill cells that express prostate cancer antigens. Using the immunocompetent TRAMP mouse model for prostate cancer, injection of tumors with a retrovirus carrying a gene for B7 resulted in the cure of $100 \%$ of the mice that were treated. In contrast, no responses were seen when the experiment was performed in nude mice, confirming the fact that a dendritic cell stimulated, $\mathrm{T}$ cell mediated mechanism was responsible for the tumor regressions. 
Another exciting, gene therapy based, in vivo protocol was described by Martin Sanda, MD, of the University of Michigan. This approach used a vaccinia virus that was carrying a gene for PSA. With the vaccinia virus itself being immunogenic, it's hoped that a non-MHC-I mediated, cytotoxic immune response against the coexpressed PSA protein will also be generated. While no efficacy data yet exists, Dr. Sanda presented some preliminary results from a phase I trial that demonstrated the safety of this approach. Similar approaches using vaccinia virus based immunotherapy has also been proposed by Hyam Levitsky, MD, and Leon Hwang, MD, of Johns Hopkins University.

Another alternative gene based strategy was proposed by Arie Belldegrun, MD, of the UCLA Medical Center. Given that the immunosuppressive cytokine TGF-beta can be found in high grade, advanced tumors, the use of antisense gene therapy to shut down the translation of TGF-beta mRNA may represent an effective way to eliminate TGF-beta and therefore reverse the immunosuppression within the tumor. Preliminary data in glioma patients and in bladder cancer cell lines have provided the proof of principle for this approach, which is currently undergoing development for prostate cancer patients.

Besides these gene therapy based approaches, the administration of monoclonal or polyclonal prostate cancer antibodies have also been proposed, either as a way to provoke a direct immunological response against the tumor, or as a way to deliver toxins directly to the tumor. Neil Bander, MD, presented data on one of the more promising antibodies, the prost $30 \mathrm{mAb}$, which is targeted to a cell surface epitope of PSMA. In a phase I study from Memorial Sloan Kettering, two out of six patients with androgen independent, metastatic cancer had clinically measurable responses to treatment with this antibody when it was conjugated to a therapeutic radioisotope.

\section{Novel radiotherapy approaches}

The development of new strategies for prostate cancer radiation therapy were reported by Mark Garzotto, MD, and David Scheinberg, MD, of the Memorial SloanKettering Cancer Center. While prostate cancer is relatively radiosensitive, local toxicity currently prevents the use of high dosages of radiotherapy that can reliably induce apotosis in all of the tumor. Additionally, radiotherapy has had little application to date on patients with disseminated disease, except in the palliative setting.

In order to improve the therapeutic index of ionizing radiation, Dr. Garzotto treated prostate cancer cell lines tetra decanol porbyl acetate (TPA), which stimulates the ceramide synthesis pathway and induces apoptosis. In combination with ionizing radiotherapy, a synergistic effect was seen both in vitro and in an orthotopic LnCAP model to increase ceramide synthesis and increase tumor apoptosis. However, the most interesting facet of this radiosensitizer is that the systemic, intrave- nous dosing of TPA did not simultaneously increase the radiosensitivity of adjacent tissues such as the rectum, since the TPA effect in normal tissue is to protect the cell from radiation induced apotosis.

Additionally, a novel method for the systemic delivery of ionizing radiation was reported by David Scheinberg, MD, of the Memorial Sloan-Kettering Cancer Center. To date, he has performed in vitro studies using the alpha particle emitting compound Bisimuth 213 (Bi 213), which was conjugated to an antibody to PSMA. In these experiments, LnCAP specific cell killing was achieved with minimal bystander toxicity seen in non PSMA expressing cells.

\section{Conclusion}

Significant progress has been made over the last year in defining the molecular mechanisms involved in the progression of prostate cancer to the androgen independent state. Through powerful, high throughput molecular biology techniques that can rapidly identify potential cancer genes, and through an evolving understanding of the mechanisms of signal transduction, hormone-receptor interactions, apoptosis pathways, and angiogenesis, new strategies are rapidly being developed for the treatment of prostate cancer (Figure 2).

While this article did not review the advances in cytotoxic chemotherapy or dietary therapies for the treatment of prostate cancer, it's clear that both approaches will also have benefits to certain patients. Continued attempts are being sponsored by CaP CURE to speed the transfer of new chemical compounds from the lab and into the clinic through the CaP CURE Therapy Consortium (Table 1). Additionally, UCLA, UCSF, the University of Wisconsin, the University of Washington, and Memor-

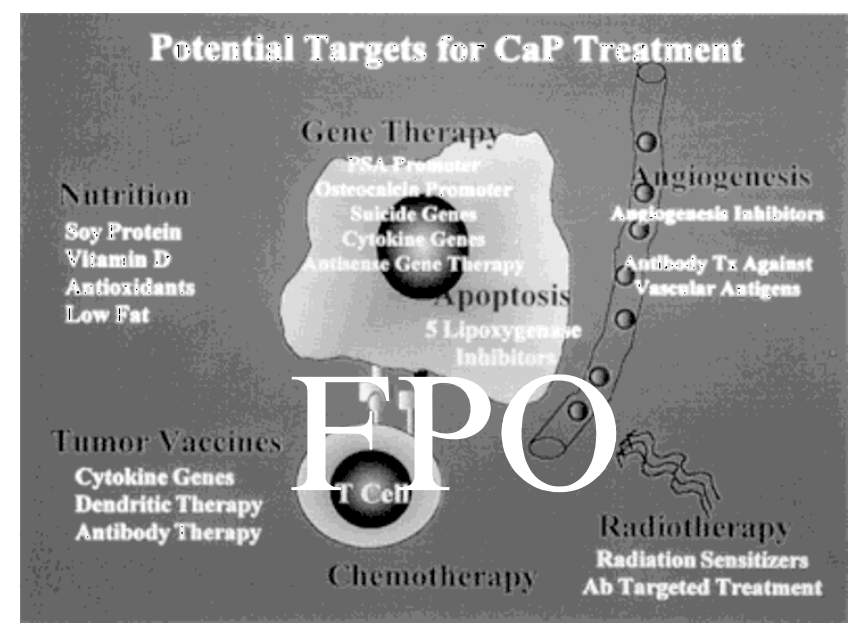

Figure 2. The new directions in the treatment of prostate cancer as defined by the 1997 CapCure Scientific Retreat. 
ial Sloan-Kettering Cancer Center are looking at the effects of dietary factors such as Vitamin D, low fat diets, and soy proteins, on the growth rate of cancer. Due to the interactions between calcium, vitamin D metabolism, and prostate cancer growth, new dietary recommendations (Figure 3) were released by the $\mathrm{CaP}$ CURE Nutrition Consortium.

In conclusion, a great deal of gratitude from the urologic research community is owed to Mike Milken, Dr. Stuart Holden, Dr. Richard Atkins, and to the entire CaP CURE staff, for not only organizing a meeting that facilitated the rapid exchange of data and new ideas, but for supporting the research that will hopefully result in the cure for advanced prostate cancer.

\section{Highlights from The CaPCURE nutrition consortium consensus statement on calcium and vitamin $D$}

\section{Calcium}

- Excessive intake of calcium (over the US RDA of $800 \mathrm{mg}$ ) from dairy products or supplements has been associated with an increased progression rate of prostate cancer.

-Protein Technologies, Inc., has agreed to reduce the calcium in their current soy formula to allow for dietary soy supplementation while keeping calcium intake to less than $400 \mathrm{mg}$ per day.

- Studies are underway to determine the role of calcium involvement in CAP progression.

\section{Vitamin D}

- Preliminary data suggest that, in addition to a possible benefit to men and women with osteoporosis, elevated vitamin D levels may be associated with a lower risk of prostate cancer.

-Vitamin D supplementation, in the recommended form of cholecalciferol or vitamin D3, should be performed under physician supervision.

The CaPCURE Monograph on Nutrition containing detail on these and other nutrition issues is available by calling the CaPCURE office at 310/458-2873.

Figure 3

Table 1 Current CaP CURE therapy consortium phase I/II clinical trials

\begin{tabular}{|c|c|c|c|}
\hline Agent $^{*}$ & Mechanism & Target population & Site \\
\hline LY 320236 & 5 alpha reductase inhibitor & Advanced Disease & $\mathrm{JHH}^{* *}$ \\
\hline LHRH Vaccine & Peptide Based Vaccine & Advanced Disease & $\mathrm{DF} / \mathrm{PCC}$ \\
\hline Torimephene & Antiestrogen & Androgen Resistant & $\mathrm{DF} / \mathrm{PCC}$ \\
\hline Emcyt/vinblastine/XRT & Chemohormonal & High Risk Localized & MSKCC \\
\hline Doxil/hormones/XRT & Chemohormonal & High Risk Localized & DF/PCC \\
\hline Emcyt/Taxol/Carboplatin & Chemotherapy & Locall Advanced and Metastatic & MSKCC and DF/PCC \\
\hline Emcyt/VP16 & Chemotherapy & Rising PSA after Primary Therapy & UMCC \\
\hline $\begin{array}{l}\text { Emcyt/Vinblastine/ } \\
\text { Ketoconazole/Adriamycin }\end{array}$ & Chemotherapy & High Risk Localized & MDA \\
\hline Suramin/Topotecan & $\begin{array}{l}\text { Chemotherapy }+ \\
\text { topo } 1 \text { inhibitor }\end{array}$ & Hormone Refractory & $\mathrm{JHH}$ \\
\hline $\begin{array}{l}\text { Emcyt/Taxol/VP16/ } \\
\text { Carboplatin }\end{array}$ & Chemotherapy & Hormone Refractory & UMCC \\
\hline Navelbine/Emcyt & Chemotherapy & Hormone Refractory & $\mathrm{DF} / \mathrm{PCC}$ \\
\hline $\begin{array}{l}\text { Strontium } 89 / \text { Emcyt/ } \\
\text { Vinblastine }\end{array}$ & $\begin{array}{l}\text { Chemotherapy }+ \\
\text { Bone Specific XRT Agent }\end{array}$ & Advanced Disease with Bone Metastasis & MDA and MSKCC \\
\hline MDX H210 & $\mathrm{Ab}$ to Her2neu & High risk patient following RP & $\mathrm{JHH}$ \\
\hline Allovax & $\begin{array}{l}\text { GM-CSF Transduced Autologous } \\
\text { Tumor Vaccine }\end{array}$ & High risk patient following $\mathrm{RP}$ & $\mathrm{JHH}$ \\
\hline C225 + Doxorubicin & EGF-R antibody + chemotherapy & Hormone Refractory & MSKCC \\
\hline PSA Vaccine (ProstVac) & Vaccinia-PSA gene Immunotherapy & Hormone Refractory & $\mathrm{DF} / \mathrm{PCC}+\mathrm{UHCC}$ \\
\hline GM-2 Conjugate/QS21 & Vaccine Against Epithelial Antigens & Hormone Refractory & MSKCC \\
\hline Vitamin D Analogues & Differentiation Agent & Hormone Refractory & UWise \\
\hline Perillyl Alcohol & Differentiation Agent & Hormone Refractory & UWise \\
\hline Phenylburtyrate & Differentiation Agent & Hormone Refractory & JHH \\
\hline ABT627 & Endothelin Antagonist & & $\mathrm{JHH}$ \\
\hline Dolastatin & Tubulin Polymerization Inhibitor & & JHH \\
\hline Flavopiridol & CDK Inhibitor & & U Wise and DF/PCC \\
\hline Diethylhomospermine & Polyamine synthesis inhibitor & & UWise \\
\hline AG3340 & Matrix Inhibitor & & UWise \\
\hline TNP 470 & Antiangiogenic Compound & Early Disease & MDA \\
\hline GBC 590 & $\begin{array}{l}\text { Galactoside Binding Pectin } \\
\text { (metastasis inhibitor) }\end{array}$ & & MDA \\
\hline
\end{tabular}

* Source: Philip Kantoff, MD. ** JHH: Johns Hopkins Hospital DF/PCC: Dana Farber Cancer Institute/Partners Cancer Care. MDA: MD Anderson Cancer Center. UVA: University of Virginia. U Wise: University of Wisconsin. MSKCC: Memorial Sloan Kettering Cancer Center. UMCC: University of Michigan Cancer Center. 